\title{
Reflexive Kompetenzen - Bedeutung und Anerkennung im Kontext erwachsenenpädagogischer Professionalisierung und Professionalität
}

\author{
Anita Pachner
}

Eingegangen: 11. Juni 2018 / Angenommen: 6. September 2018 / Online publiziert: 27. September 2018 (C) Der/die Autor(en) 2018

Zusammenfassung Der Beitrag fokussiert auf sogenannte Reflexive Kompetenzen, ihre Bedeutung im Kontext erwachsenenpädagogischer Professionalisierung und Professionalität sowie ihre Einbettung in bestehende Zertifizierungs- und Anerkennungsverfahren. Reflexive Kompetenzen sind ein zentraler Bestandteil erwachsenenpädagogischer Professionalität. Ihre Anerkennung und Zertifizierung ist demgegenüber jedoch besonders herausfordernd, da nicht auf detaillierte Beschreibungen und Ausformulierungen dieser Kompetenz aufgebaut werden kann. Es soll daher der Frage nachgegangen werden, wie die Anerkennung und Formulierung Reflexiver Kompetenzen bisher erfolgt und künftig auf empirischer Grundlage weiterentwickelt werden kann. Dazu wird nachgezeichnet, wo Reflexive Kompetenzen bereits früh in der Diskussion um erwachsenenpädagogische Professionalität und Professionalisierung verortet sind. Anschließend werden zwei bedeutsame Zertifizierungs- und Anerkennungsverfahren aus dem deutschsprachigen Raum vorgestellt und dabei besonders die Verortung Reflexiver Kompetenzen in den zugehörigen Kompetenzmodellen beleuchtet. Schließlich werden erste Ergebnisse eines laufenden Forschungsprojekts präsentiert, welches die Rekonstruktion und Formulierung der Kompetenz zur (Selbst-)Reflexion zum Ziel hat. Anhand einer exemplifizierenden Einzelfalldarstellung wird ein vertiefender Einblick in die bestehende Praxis eines etablierten Verfahrens zur Anerkennung Reflexiver Kompetenzen gewährt. So können Chancen und Grenzen dieses Verfahrens für die Rekonstruktion Reflexiver Kompetenzen ausgelotet werden und Schlussfolgerungen für eine empirisch fundierte Weiterentwicklung gezogen werden.

Dr. A. Pachner $(\bowtie)$

Institut für Erziehungswissenschaft, Abteilung Erwachsenenbildung/Weiterbildung, Eberhard Karls Universität Tübingen, 72070 Tübingen, Deutschland

E-Mail: anita.pachner@uni-tuebingen.de 
Schlüsselwörter Reflexive Kompetenzen · Kompetenzvalidierung · Pädagogische Professionalität · Professionalisierung · Erwachsenenbildung

\title{
Reflexive competencies-importance and recognition in the context of adult educational professionalization and professionalism
}

\begin{abstract}
The article focuses on reflexive competencies, their importance in the context of adult educational professionalization and professionalism, and their embedding in existing procedures for the validation of competencies. Reflexive competencies are a key component of adult educational professionalism. Their recognition and certification, however, is particularly challenging because it cannot be based on detailed descriptions of these competencies. Therefore, it will be examined how the recognition and formulation of reflexive competencies has been done so far and can be further developed on an empirical basis in the future. First, it will be shown that reflexive competencies are early situated in the discussion of adult educational professionalism and professionalization. Subsequently, two current procedures for the certification and recognition of competencies from German-speaking countries will be presented, highlighting in particular the embedding of reflexive competencies in the corresponding competency models. Finally, first results of an ongoing research project are presented. This project aims at the reconstruction and formulation of the (self-)reflection competence. On the basis of an exemplary case study, a deeper insight is given into the existing practice of an established procedure for the recognition and validation of reflexive competencies. Thus, the strengths and weaknesses of this procedure for the reconstruction of reflexive competencies can be explored and conclusions can be drawn on how to further develop it on an empirical basis.
\end{abstract}

Keywords Self-reflection competence $\cdot$ Validation of competencies · Pedagogic professionalism $\cdot$ Professionalization $\cdot$ Adult education

\section{Die Anerkennung und Formulierung Reflexiver Kompetenzen - eine aktuelle Herausforderung}

Die Formulierung und Anerkennung von Kernkompetenzen stellt in der Erziehungswissenschaft nach wie vor eine ungelöste Problemstellung dar. Dies gilt vor allem, wenn soziale oder personale Kompetenzen differenziert beschrieben werden sollen oder es um die Validierung non-formal und informell erworbener Lernergebnisse und Kompetenzen geht. Sie zeichnen sich durch einen besonders hohen Grad an Komplexität und Subjektivität aus. Dementsprechend fordern Gruber und Wiesner (vgl. 2012, S. 14f.) verstärkte Anstrengungen zu unternehmen, um dem Dreischritt aus Formulierung, Anerkennung und schließlich Vermittlung von Kernkompetenzen nachzukommen. Alle drei Aspekte haben wichtige Auswirkungen für die Professionalisierung und Professionalität pädagogischen Personals.

Im Professionalisierungsdiskurs der deutschsprachigen Erwachsenenbildung gewinnt die Anerkennung von auf non-formalen oder informellen Wegen erworbenen Kompetenzen an Bedeutung (vgl. z.B. das BMBF-Projekt GRETA am DIE). Dies 
trägt zum einen Forderungen Rechnung, wie sie im Deutschen Qualifikationsrahmen (AK DQR 2011) festgehalten sind oder aus der EU-Empfehlung hervorgehen, der zufolge bis zum Jahr 2018 entsprechende nationale Validierungsstrategien entwickelt werden sollten (Cedefop 2015, S. 4). Zum anderen knüpft es an die bereits bewährte Praxis in den deutschsprachigen Nachbarländern an, wie sie z. B. bei der Weiterbildungsakademie Österreich (wba) oder der SVEB in der Schweiz ausgeübt wird.

Im vorliegenden Beitrag soll der Fokus auf Reflexiven Kompetenzen, ihrer Bedeutung im Kontext erwachsenenpädagogischer Professionalisierung und Professionalität sowie ihrer Einbettung in bestehende Zertifizierungs- und Anerkennungsverfahren liegen. Reflexive Kompetenzen sind seit jeher ein wichtiger Bestandteil erwachsenenpädagogischer Professionalität und damit auch der Professionalisierung in diesem Bereich. Gerade bei der ,Bewältigung komplexer, divergenter Situationen und Handlungsanforderungen“, wie sie heute kennzeichnend für berufliches Handeln sind, kommt ihnen zentrale Bedeutung zu (Pachner 2013, S. 5; Schön 1987, S. 35). Ihre Anerkennung und Zertifizierung ist demgegenüber jedoch besonders herausfordernd, da nicht auf detaillierten Beschreibungen und Ausformulierungen dieser Kompetenzen aufgebaut werden kann. Es soll daher der Frage nachgegangen werden, wie die Anerkennung und Formulierung Reflexiver Kompetenzen bisher erfolgt und künftig auf empirischer Grundlage weiterentwickelt werden kann.

Im Folgenden wird deshalb zunächst nachgezeichnet, wo Reflexive Kompetenzen bereits früh in der Diskussion um Professionalität und Professionalisierung vorkommen und mit welchen Ansätzen erwachsenenpädagogische Professionalität daraufhin untersucht werden kann. Anschließend werden zwei bedeutsame Zertifizierungsund Anerkennungsverfahren aus dem deutschsprachigen Raum vorgestellt und dabei besonders die Verortung Reflexiver Kompetenzen in ihnen beleuchtet. Schließlich werden erste Ergebnisse eines laufenden Forschungsprojekts vorgestellt, welches die Rekonstruktion und Formulierung der Kompetenz zur (Selbst-)Reflexion zum Ziel hat (vgl. z.B. Pachner 2014). Dies geschieht anhand einer exemplifizierenden Falldarstellung. Sie ermöglicht es, einen vertieften Einblick in die bestehende Praxis eines etablierten Verfahrens zur Anerkennung Reflexiver Kompetenzen zu gewinnen sowie Chancen und Grenzen dieses Verfahrens für die Rekonstruktion Reflexiver Kompetenzen auszuloten. Im Mittelpunkt stehen dabei Lehrende in der Erwachsenenbildung. Der Beitrag schließt mit einem Ausblick auf Potenziale des vorgestellten Forschungsprojekts, auch die Formulierung und Vermittlung Reflexiver Kompetenzen weiterzuentwickeln und damit forschungsmethodisch wie praktisch einen wichtigen Beitrag zur Professionalität und Professionalisierung in diesem zentralen Bereich erwachsenenpädagogischer Kompetenz zu leisten. 


\section{Reflexive Kompetenzen im Kontext erwachsenenpädagogischer Professionalisierung und Professionalität - Theoretische Verortung}

\subsection{Begriffliche Einordnung}

Der Begriff „Professionalität“ nimmt die Handlungsperspektive in den Blick. Gegenstand von Analysen ist dementsprechend die konkrete Handlungssituation. Dabei ist Professionalität immer nur als flüchtiger Zustand zu betrachten, der interaktiv immer wieder neu hergestellt werden muss und daher ein hohes Maß an Reflexivität und Begründungsfähigkeit seitens der professionellen Akteure verlangt (vgl. Nittel 2011, S. 48). Für die Erwachsenenbildung formulieren Gruber und Wiesner (2012, S. 14) folglich:

Erwachsenenpädagogische Professionalität basiert - neben einer theoriebegründeten und handlungsorientierten Wissensbasis - auf Deutungen, Diagnosen und Interpretationen, sie ist nicht etwas Abgeschlossenes, sondern sie muss sich als Kompetenz immer wieder bewähren und neu entwickeln (vgl. Nittel 2000, S. 85). Somit setzt dies über wissenschaftliches Grundlagenwissen hinaus vor allem praktische Erfahrungen und ein hohes Maß an Reflexionsfähigkeit voraus.

Damit ist die große Bedeutung, die Reflexive Kompetenzen neben Wissen und Praxiserfahrungen für professionelles Handeln haben, angesprochen. Die Verwendung der Begrifflichkeit „Reflexive Kompetenzen“ fußt im Folgenden auf einem Verständnis von (Selbst-)Reflexion, das in Anlehnung an Greif (2008, S. 40) als „ergebnisorientierte Selbstreflexion“ bezeichnet werden kann.

Sie ist definiert als ein bewusster Prozess, ,,bei dem eine Person ihre Vorstellungen oder Handlungen durchdenkt und expliziert, die sich auf ihr reales und ideales Selbstkonzept beziehen. Ergebnisorientiert ist die Selbstreflexion, wenn die Person dabei Folgerungen für künftige Handlungen oder Selbstreflexionen entwickelt" (Greif 2008, S. 40).

\subsection{Das kompetenz- und das differenztheoretische Verständnis von Professionalität}

Professionalität kann kompetenztheoretisch oder differenztheoretisch aufgefasst werden. Der kompetenztheoretische Ansatz interessiert sich für die Fertigkeiten und Fähigkeiten, die seitens der beruflichen Rollenträger erforderlich sind, um eine bestimmte, berufliche Aufgabe zu erfüllen. Der differenztheoretische Ansatz nimmt dagegen die Kernprobleme, Dilemmata und Widersprüche im professionellen Handeln in den Blick. Schütze (1996), ein prominenter Vertreter einer am Symbolischen Interaktionismus orientierten Perspektive auf Professionalität, folgt diesem Definitionsansatz und der entsprechenden Fokussierung bei der Erforschung konkreten professionellen Handelns. Kritisch merkt Nittel (2011, S. 49f.) dazu an, dass die identifizierten Kernprobleme nicht weiter systematisiert werden. Eine Unterscheidung nach verschiedenen Gattungen von Paradoxien in z. B. allgemeine versus bereichsbezogen wirksame bleibt ebenso aus wie Aussagen darüber fehlen, 
ob diese Kernprobleme gerade die Voraussetzung für die Begründung von Professionalität darstellen oder ihr Zustandekommen vielmehr verhindern und wie mit ihnen umgegangen wird bzw. werden kann. Diesen Anspruch löst von Hippel (2011) ein, wenn sie die Differenzierung nach Antinomien, Widerspruchskonstellationen und Paradoxien von Helsper (2002) und die damit verbundene Begründungslogik für die Erwachsenenbildung und dabei insbesondere das Programmplanungshandeln erschließt. Gleichzeitig stellt sie fest: „Die Analyse von Widerspruchskonstellationen und Antinomien im mikrodidaktischen, lehrenden Aufgabenbereich stellt noch ein Desiderat dar“ (von Hippel 2011, S. 45). Dabei sind solche Widerspruchskonstellationen nicht nur negativ zu sehen, sondern bergen - bei gelingender, ,reflexiver Handhabung“ - auch das Potenzial für ,Dynamik und Produktivität“, indem sie ,alternative Gestaltungsmöglichkeiten“ in den Blick bringen (von Hippel 2011, S. 46; vgl. auch Pachner 2013).

Wenn es im Folgenden um die Anerkennung von non-formal und informell erworbenen Kompetenzen bei Lehrenden in der Erwachsenenbildung geht, scheint es naheliegend, dass ein kompetenztheoretisches Verständnis von Professionalität eher zugrunde gelegt werden sollte als ein differenztheoretischer Blick auf den Gegenstand. Das ist mit Bezug auf (selbst-)reflexive Kompetenzen nicht unbedingt der Fall. Beim kompetenztheoretischen Verständnis von Professionalität wird professionelle Kompetenz als Disposition zur Erbringung einer beruflichen Leistung verstanden (vgl. Nittel 2000, S. 74; Erpenbeck und von Rosenstiel 2007). Professionalität - also der sichtbare Beweis dafür, dass ebenjene Leistung erbracht wurde - könnte dann als die zugehörige Performanz beschrieben werden. Kompetenzprofile oder -kataloge umschreiben verschiedene Elemente und Teilaspekte der beruflichen Kompetenz. Als wesentliche Bereiche erwachsenenpädagogischer Kompetenz wurden der Umgang mit der Klientel und den Teilnehmenden im direkten Kontakt, das Handeln in Organisationen, der Umgang mit sich selbst und das Vermitteln von Inhalten identifiziert. Als Kernkompetenzen, die sich an den grundlegenden Handlungsformen von in der Erwachsenenbildung Tätigen orientieren, werden dementsprechend Unterrichten, Beraten und Organisieren genannt (vgl. Nittel 2000, S. 76ff.). Sofern diese Kompetenzen und Kompetenzbereiche alle angesprochen sind, kann ein Kompetenzkatalog als vollständig gelten. Reflexive Kompetenzen treten dabei bereits früh und in unterschiedlichen Wendungen in Erscheinung. So nennt Siebert (1990, S. 285) als eine Dimension professioneller Kompetenz:

Eine reflexive Sensibilität, d.h. eine Vergewisserung und Begründung der eigenen Motive und Interessen, Stärken und Schwächen, der professionellen und institutionellen Möglichkeiten und Grenzen, der Auswirkungen des eigenen Handelns und Verhaltens auf Mitarbeiter-/innen, Adressaten und Teilnehmer/ -innen, die Klärung der eigenen pädagogischen Identität.

Glagow u.a. (zit. nach Dewe 1990, S. 294) führen als eine generalisierbare Komponente pädagogischer Handlungskompetenz ebenfalls reflexive Kompetenz mit auf und zwar:

die Fähigkeit zur sensiblen Selbstkontrolle und -beschränkung im Rahmen unterrichtlicher ... Entscheidungen sowie Lehr-/Lernmethodenwahl. Bei dieser $r e-$ 
flexiven Kompetenz geht es um Formen der Abwägung und Ausrichtung des Handelns hinsichtlich seiner längerfristigen pädagogischen und qualifikatorischen Folgen.

Kritisiert werden kann an kompetenztheoretischen Ansätzen, dass lediglich das „Potential von Professionalität“ untersucht wird, nicht jedoch die Professionalität selbst, dass sie gleichsam „zur Seite des faktischen Berufshandelns hin blind sind“ (Nittel 2000, S. 79). Dies bedeutet jedoch nicht, dass der kompetenztheoretische Ansatz gänzlich zu verwerfen wäre, denn, so räumt Nittel (2000, S. $80 \mathrm{ff}$.) ein, verkörpere er ,wie kein anderer die pädagogische Denkungsart, welche durch die Spannung zwischen einer wirklichen und einer möglichen pädagogischen Praxis gekennzeichnet ist“".

Von einem kompetenztheoretischen Ansatz ist das differenztheoretische Verständnis von Professionalität zu unterscheiden. Es stellt die Spannungsverhältnisse, die die pädagogische Berufsarbeit kennzeichnen, in den Mittelpunkt der Aufmerksamkeit. Zentral ist hierbei die Schwierigkeit, zwischen Theorie und Praxis vermitteln zu sollen. Nittel (2000, S. 81 ff.) merkt an, dass dieses Dilemma nach drei Seiten hin aufgelöst werden kann: zur Handlungs-, zur Wissens- und zur Beziehungsebene. Auf jeder dieser drei Ebenen ergeben sich wiederum je spezifische Widersprüchlichkeiten. Auf der Handlungsebene sind die professionell Tätigen aufgerufen, zwischen Entscheidungsdruck in der konkreten Situation und einer hohen Begründungsleistung für das professionelle Tun zu balancieren. Auf der Wissensebene sind unterschiedliche, gleichzeitig wirksame Wissensbestände aus Professionswissen, Alltagswissen, wissenschaftlichem Wissen und Handlungswissen produktiv zu nutzen. Auf der Ebene der Beziehung zwischen professioneller Erwachsenenbildnerin bzw. professionellem Erwachsenenbildner und Klienten bzw. Teilnehmenden ist die widersprüchliche Gleichzeitigkeit von Nähe und Distanz, von funktional spezifischen und funktional diffusen Momenten auszuhalten. Professionalität kann unter diesen Bedingungen definiert werden ,als eine gelungene Form der praktischen Bearbeitung dieser spannungsreichen Konstellationen ..., wobei unter ,gelungen " nicht mehr und nicht weniger verstanden wird als die Vermeidung von Vereinseitigungen" (Nittel 2000, S. 84). Professionalität äußert sich schließlich

auf der Ebene der professionellen Selbstdeutung ..., oder anders ausgedrückt: Der Erwachsenenbildner agiert nicht nur „gut“, er weiß auch, dass er „gut“ ist. Professionalität beinhaltet einen Reflexionsstil und Urteilsformen, die dem Akteur hohe Begründungsleistungen abverlangen und eine realistische Selbstbeobachtung ermöglichen (Nittel 2000, S. 84).

Insbesondere diese differenztheoretische Perspektive auf Professionalität ist für die Analyse Reflexiver Kompetenzen heuristisch wertvoll, wie sich im vierten Abschnitt bei der Vorstellung erster empirischer Befunde aus einer sich noch in Arbeit befindenden Studie zeigen wird. Gerade der professionelle Umgang mit widersprüchlichen Handlungsanforderungen fordert Reflexive Kompetenzen heraus und birgt damit auch Chancen für Weiterentwicklung und Veränderung (vgl. Pachner 2013; von Hippel 2011, S. 45 f.). Ergänzend dazu darf der Verweis nicht ausbleiben, dass bei der Analyse von non-formal oder informell erworbenen Kompetenzen 
Lehrender in der Erwachsenenbildung besonders Prozesse einer ,individuellen Professionalisierung" interessant sind.

Nittel und Seltrecht (2008, S. 124) definieren individuelle Professionalisierung als

einen an das konkrete Individuum gebundenen Ausbildungs- und Reifeprozess, der nicht zwingend an eine wissenschaftliche Ausbildung gebunden sein muss, aber dennoch zu einem Statuserwerb und zu einer pädagogisch-professionellen Praxis sowie zu einem diesbezüglichen Selbstbild führt.

\section{Reflexive Kompetenzen und ihre Anerkennung in bestehenden Zertifizierungsverfahren - Praxisbezogene Verortung an zwei Beispielen}

\subsection{Die Zertifizierungs- und Kompetenzanerkennungsstelle Weiterbildungsakademie Österreich (wba)}

\subsubsection{Entstehung und Ablauf des Zertifizierungsverfahrens}

Die Weiterbildungsakademie Österreich (wba) hat am 01. Februar 2007 als Zertifizierungs- und Kompetenzanerkennungsstelle ihre Arbeit aufgenommen. Sie geht zurück auf ein gemeinsames ESF-Projekt (2004-2006) der zehn größten Erwachsenenbildungsverbände Österreichs, der Konferenz der Erwachsenenbildung Österreich (KEBÖ) und dem Bundesinstitut für Erwachsenenbildung (bifeb). Koordiniert wurde das Projekt vom Verband Österreichischer Volkshochschulen. Getragen wird die wba vom Kooperativen System der österreichischen Erwachsenenbildung am bifeb. Es ist verantwortlich für die pädagogischen und strategischen Angelegenheiten der wba. Sein Ziel ist die Professionalisierung und Qualitätsentwicklung der österreichischen Erwachsenenbildung (www.wba.or.at). Seit Gründung der wba wurden insgesamt 1857 Standortbestimmungen abgeschlossen und 1142 wba-Zertifikate sowie 235 wba-Diplome erreicht (https://wba.or.at/de/ueber-uns/daten-undfakten.php).

Aufbauend auf einer „Standortbestimmung“ kann die Kompetenzzertifizierung mit zwei aufeinander aufbauenden, landesweit gültigen Zertifikaten abgeschlossen werden, dem wba-Zertifikat und dem wba-Diplom, für welches zudem einer der vier Schwerpunkte Lehren/Gruppenleitung/Training, Bildungsmanagement, Beratung oder Bibliothekswesen zu wählen ist. Für die Standortbestimmung sind alle formal, non-formal oder informell erworbenen Kompetenzen in ein Online-Portfolio einzutragen und mit entsprechenden Nachweisen unterschiedlichster Art zu belegen. Die Standortbestimmung orientiert sich an einem eigens entwickelten Kompetenzmodell, dem „Qualifikationsprofil““. Für alle erwerbbaren Zertifikate liegt ein eigenes Qualifikationsprofil vor. Gemeinsam mit einer wba-Beraterin wird nach Rückmeldung der Ergebnisse der Standortbestimmung aus dem Akkreditierungsrat ein Bildungsplan entwickelt. Sind offene Kompetenzen nachzuholen, geschieht dies über von der wba akkreditierte Bildungsangebote. Nach erfolgreichem Ab- 
schluss eines mehrtägigen Assessmentcenters, der „Zertifizierungswerkstatt“, kann der Abschluss „Zertifizierte/r Erwachsenenbildner/in“ erworben werden. Mit dem Abschluss „Diplomierte/r Erwachsenenbildner/in“ kann der Expertenstatus in einem oder mehreren der vier genannten Schwerpunkte vorgewiesen werden. Zusätzlich ist eine Diplomarbeit vorzulegen, die auch dem Nachweis Reflexiver Kompetenz dient. Der Zertifizierungsprozess schließt mit einem Prüfungskolloquium ab. Das wba-Diplom öffnet den Weg zu universitären Weiterbildungslehrgängen (https://wba.or.at/ de/leistungen).

\subsubsection{Reflexive Kompetenzen in den Kompetenzprofilen der wba}

Die den Zertifizierungsverfahren zugrunde liegenden Kompetenzen sind in sogenannten Qualifikationsprofilen zusammengefasst. Sie basieren auf den Anforderungen, denen sich professionelle Praktiker und Praktikerinnen der Erwachsenenbildung in ihrem Berufsalltag gegenübersehen. Die Qualifikationsprofile umfassen dabei ,einerseits generelle, sehr breit gefächerte Kenntnisse, Fertigkeiten und Kompetenzen, andererseits spezialisierte, tief greifende“ (wba-Zertifikat 2018, S. 4). Aufgebaut sind die wba-Qualifikationsprofile dergestalt, dass sie die geforderten Kompetenzbereiche samt Teilkompetenzen beschreiben. Jedem Kompetenzbereich sind Pflichtund Wahlteile zugeordnet und mit hierfür zu vergebenden ECTS (wba) gewichtet. Zudem werden Formen möglicher Nachweise für die geforderten Kompetenzen benannt.

Für das wba-Zertifikat sind 60 ECTS (wba) nachzuweisen, die sich auf sieben Kompetenzbereiche zuzüglich Standortbestimmung, Allgemeine Wahlmodule, Zertifizierungswerkstatt und Verpflichtende Praxis verteilen (wba-Zertifikat 2018, S. 2 ff.). Die Fähigkeit zu reflektiertem Handeln kommt als Querschnittskompetenz in verschiedenen Kompetenzbereichen des wba-Zertifikats vor. Zentralen Stellenwert nimmt sie im Kompetenzbereich 8 „Personale Kompetenz“ ein (wba-Zertifikat 2018, S. 24f.). Das ist eine durchaus gängige Verortung. Sie findet sich so z.B. auch im Deutschen Qualifikationsrahmen (DQR), in dem „Reflexivität“ als Teil der Personalen Kompetenz im Bereich ,Selbstständigkeit“ aufgeführt ist und wie folgt definiert wird: „Reflexivität beinhaltet die Fähigkeit, mit Veränderungen umzugehen, aus Erfahrungen zu lernen und kritisch zu denken und zu handeln“ (AK DQR 2011, S. 9). Personale Kompetenz und insbesondere die Fähigkeit zur Selbstreflexion wird im Qualifikationsprofil für das wba-Zertifikat aus der folgenden beruflichen Anforderung abgeleitet: „Sie sind sich ihrer vielen unterschiedlichen beruflichen Rollen bewusst und sind bereit und fähig, ihr Handeln zu reflektieren. Das Vermögen zur Selbstreflexion ist ein hoher Wert und ein professionelles Merkmal bei allen Erwachsenenbildner/innen“ (wba-Zertifikat 2018, S. 24).

Verpflichtende Inhalte sind dementsprechend eine „Stärken- und Schwächenanalyse“ sowie die „Analyse und Reflexion eigenen Handelns und Verhaltens in erwachsenenbildungsrelevanten Zusammenhängen (im Rahmen von Lehrgängen oder Ausbildungen)“ (wba-Zertifikat 2018, S. 24). Bescheinigungen über den Besuch solcher Lehrgänge können als Nachweise angeführt werden, wozu auch die Inanspruchnahme von Supervision und Coaching zählen, und zusätzlich der erfolgreiche Besuch der Zertifizierungswerkstatt. Auf den Punkt gebracht sollen die zertifizier- 
ten Erwachsenenbildnerinnen und Erwachsenenbildner in der Lage sein, „den Blick auf ihr professionelles Handeln und Verhalten zu lenken, dieses zu analysieren, zu reflektieren und Schlussfolgerungen daraus zu ziehen“ (wba-Zertifikat 2018, S. 24). Dies kommt der Auffassung einer ,ergebnisorientierten“ Selbstreflexion recht nahe, wie sie oben in Anlehnung an Greif (2008) bereits definiert wurde. Ihr zentrales Kennzeichen ist es, dass die Reflexion in Folgerungen für künftiges Denken, Reflektieren und Handeln mündet.

Im Qualifikationsprofil für das wba-Diplom hier am Beispiel des Schwerpunkts Lehren/Gruppenleitung/Training werden 14 Kompetenzbereiche mit insgesamt 60 ECTS (wba) verlangt. Reflexive Kompetenz taucht in verschiedenen Kompetenzbereichen auf, so auch hier im Bereich „Personale Kompetenz“. Mit dem Kompetenzbereich 8 ,Reflexive Kompetenz: Diplomarbeit“ erhält sie zudem einen zentralen, eigenen Ort. Dieser ist nach dem Bereich „Verpflichtende Praxis“ und dem Bereich „Didaktische Kompetenz“ der am dritthöchsten gewichtete im wba-Diplom (wba-Diplom 2016, S. 2). Der Bereich „Personale Kompetenz“ enthält gegenüber dem wba-Zertifikat zusätzlich Aspekte der Selbstregulation und der motivationalen Orientierungen (vgl. z. B. Baumert und Kunter 2006). Neu hinzu kommt beim wbaDiplom, dass die Reflexive Kompetenz ,tätigkeitsbegleitend“ und mit Bezugnahme auf relevante Theorien für jeden der vier Bereiche nachgewiesen werden muss (wbaDiplom 2016, S. 19). Dazu ist eine Diplomarbeit anzufertigen, in der zum einen ein praxisnahes Thema kritisch reflektiert werden soll und zum anderen die eigene Rolle, das eigene Arbeitsverhalten und die eigene Arbeitshaltung. Dies soll mittels Selbst- und ggf. Fremdwahrnehmung geschehen, wobei nicht näher ausgeführt wird, wie diese Selbstwahrnehmung zu vollziehen ist. Als Kern der Reflexiven Kompetenz sollen die diplomierten Erwachsenenbildner und Erwachsenenbilderinnen in der Lage sein,

ihre erwachsenenbildnerische Tätigkeit, ihre Position, Aufgaben und ihr Rollenverständnis darzustellen, Stärken und Schwächen ihres professionellen Handelns und Verhaltens kritisch zu erfassen und professionelle wie persönliche Entwicklungsmöglichkeiten aufzuzeigen (wba-Diplom 2016, S. 19).

Auch dieses Verständnis von (Selbst-)Reflexion ähnelt der Definition von Greif (2008). Und es werden wie bei Nittel (2000, S. 84) „hohe Begründungsleistungen“ und eine „realistische Selbstbeobachtung“ als Kennzeichen erwachsenenpädagogischer Professionalität verlangt.

\subsection{Das Anerkennungsverfahren des GRETA-Projekts am DIE}

Ein vergleichbares Vorhaben wurde in Deutschland mit dem BMBF-geförderten und vom Deutschen Institut für Erwachsenenbildung - Leibniz-Zentrum für Lebenslanges Lernen (DIE) geleiteten Projekt GRETA (Dezember 2014 bis November 2018) begonnen (www.die-bonn.de/greta). GRETA steht für „Grundlagen für die Entwicklung eines trägerübergreifenden Anerkennungsverfahrens für die Kompetenzen Lehrender in der Erwachsenen- und Weiterbildung“. Zentrales Ziel des Projekts ist die Anerkennung von Kompetenzen Lehrender in der Erwachsenenbildung/Weiterbildung und damit die weitere Professionalisierung dieser Gruppe. 
Voraussetzung für die Entwicklung eines Verfahrens zur Anerkennung von Kompetenzen war auch hier die Formulierung eines Kompetenzmodells, das alle für eine Lehrtätigkeit in der Erwachsenenbildung wichtigen Kompetenzen umfasst. Damit ein solches Kompetenzmodell möglichst seitens aller Nutzergruppen Akzeptanz erfährt, ist deren Einbezug bereits in der Entwicklungsphase sinnvoll. So wurde auch das GRETA-Kompetenzmodell in enger Zusammenarbeit von Wissenschaft und Praxis entwickelt (Lencer und Strauch 2016). Kooperationspartner aus der Praxis waren ähnlich wie bei der wba in Österreich acht Dachverbände und -organisationen zentraler Träger der Erwachsenenbildung in Deutschland (DIE 2018, S. 7). In einem ersten Schritt wurden gemeinsam Verfahren und geeignete Instrumente für die Validierung auch non-formal und informell erworbener Kompetenzen konzipiert, die sich gegenwärtig in der Erprobung befinden. Gleichzeitig bietet das Kompetenzmodell den Ausgangspunkt für einen zweiten Weg der Anerkennung von Kompetenzen. Über ein sogenanntes Mapping können Bildungsanbieter ihre Train-the-Trainer-Seminare mit dem GRETA-Kompetenzmodell abgleichen und das Ergebnis des Mappings künftig auf einer Plattform speichern (DIE 2018, S. 5). Dies soll Lehrenden der Erwachsenenbildung die Suche nach geeigneten Angeboten zur Vervollständigung ihres Kompetenzprofils erleichtern und sie so in ihrer weiteren Professionalisierung unterstützen.

Für die eigentliche Bilanzierung und Anerkennung der non-formal und informell erworbenen Kompetenzen der Lehrenden wurde auf Basis des GRETA-Kompetenzmodells das Validierungsinstrument „PortfolioPlus“ entwickelt. Darin können die Lehrenden ihre Kompetenzen dokumentieren, durch geschulte Gutachter bewerten lassen und in eine „Kompetenzbilanz“ überführen lassen. Diese wird in einem Beratungsgespräch an die Lehrenden zurückgemeldet (DIE 2018, S. 4).

Das GRETA-Kompetenzmodell fußt dabei auf einem umfassenden Verständnis von Kompetenzen, wie es Weinert (2001, S. 27) formuliert:

Unter „Kompetenzen“ versteht er die

bei Individuen verfügbaren oder durch sie erlernbaren kognitiven Fähigkeiten und Fertigkeiten, um bestimmte Probleme zu lösen, sowie die damit verbundenen motivationalen, volitionalen und sozialen Bereitschaften und Fähigkeiten, um die Problemlösungen in variablen Situationen erfolgreich und verantwortungsvoll nutzen zu können.

Dementsprechend gliedert sich das GRETA-Kompetenzmodell in die folgenden vier „Kompetenzaspekte“: Berufspraktisches Wissen und Können, Fach- und feldspezifisches Wissen, Professionelle Selbststeuerung sowie Professionelle Werthaltungen und Überzeugungen (Lencer und Strauch 2016, S. 5). Die Kompetenzaspekte umfassen jeweils zwei bis fünf Kompetenzbereiche und diese wiederum zwei bis vier Kompetenzfacetten. Die Reflexive Kompetenz, hier „Reflexion des Lehrhandelns“ genannt, findet sich neben „Umgang mit Feedback/Kritik“ und „Berufliche Weiterentwicklung“ im Kompetenzbereich „Berufspraktische Erfahrung“. Dieser bildet zusammen mit „Selbstregulation“ und „Motivationaler Orientierung“ die Kompetenzfacette „Professionelle Selbststeuerung“ (Lencer und Strauch 2016, S. 6f.). Damit, so könnte man schlussfolgern, kommt der Reflexiven Kompetenz im GRETA-Kompetenzmodell bislang eine relativ untergeordnete Stellung zu. Weiter ausgearbeitet 
werden soll das Modell künftig in Bezug auf Niveaustufen der genannten Kompetenzen, die an den Deutschen Qualifikationsrahmen (DQR) anschlussfähig sein sollen, und bzgl. Entwicklungsperspektiven für die Weiterentwicklung der Kompetenzen von Lehrenden in der Erwachsenen- und Weiterbildung (Lencer und Strauch 2016, S. 6).

\subsection{Eine knappe Synopse}

Beide Anerkennungsverfahren - das der wba wie das aus dem GRETA-Projekt nehmen ihren Ausgangspunkt in eigens entwickelten Kompetenzprofilen. In einem ersten Schritt erfolgt ein Abgleich der bis dato durch die Praktikerinnen und Praktiker der Erwachsenenbildung entwickelten Kompetenzen mithilfe eines Portfolios. Begleitet durch eine Beraterin wird so der aktuelle Kompetenzstand im Vergleich zum zugrundeliegenden Kompetenzprofil bilanziert, also ein Soll-Ist-Vergleich vorgenommen. Die sich daran anschließenden Schritte unterscheiden sich bei beiden Verfahren deutlich, was vor allem mit dem unterschiedlichen Entwicklungsstand und Reifegrad der beiden Anerkennungsverfahren zusammenhängen dürfte. Auch die Einordnung und Bedeutung Reflexiver Kompetenzen im jeweiligen Kompetenzprofil differiert stark. Im Qualifikationsprofil der wba nehmen diese einen zentralen Stellenwert ein. Mit der wba-Diplomarbeit gibt es zudem eine eigene „Nachweisform“, welche im Wesentlichen zur Anerkennung Reflexiver Kompetenzen dient. Reflexive Kompetenz wird als eine zentrale Zielkategorie erwachsenenpädagogischer Professionalität und Professionalisierung verortet. Im GRETA-Kompetenzmodell dagegen wird die „Reflexion des Lehrhandelns“ eher aufgefasst als Mittel zur Ausbildung von Handlungskompetenz, erhält also einen nachgeordneten Stellenwert. $\mathrm{Ob}$ und wie sich ihre Anerkennung ggf. weiter ausdifferenzieren wird, werden die nächsten Arbeitsschritte im GRETA-Projekt zeigen.

\section{Die Anerkennung Reflexiver Kompetenzen - Empirische Befunde}

\subsection{Offene Fragestellungen}

Aus den bisher beschriebenen Verortungen Reflexiver Kompetenz lassen sich offene Fragestellungen ableiten, die die Anerkennung und Zertifizierung dieser meist nonformal und informell erworbenen Kompetenz betreffen. Relevante Fragestellungen in diesem Zusammenhang sind:

- Worin besteht der von Nittel (2000, S. 84) als Ausdruck erwachsenenpädagogischer Professionalität postulierte „Reflexionsstil“ und welche Art von „,professioneller Selbstdeutung“ ist hier aufschlussreich?

- Was tun die Praktikerinnen und Praktiker der Erwachsenenbildung, wenn sie über ihr professionelles Handeln kritisch reflektieren sollen und wie tun sie es?

- Oder anders formuliert: Was macht die kritische Reflexion zur Reflexion im Unterschied zur folgenlosen Beschreibung von Erfahrungen aus der Praxis und damit im Kontext Reflexiver Kompetenz anerkennenswert? 


\subsection{Einsichten in den Anerkennungsprozess - Das methodische Vorgehen}

Um einen vertieften Einblick in die bestehende Praxis der Anerkennung Reflexiver Kompetenzen zu gewinnen und gleichzeitig eine Annäherung an die genannten Fragen zu erzielen, soll auf Ergebnisse eines Forschungsprojekts zurückgegriffen werden, das es zum Ziel hat, die Kompetenz zur (Selbst-)Reflexion aus Aussagen von Erwachsenenbildnerinnen und Erwachsenenbildnern praxisnah zu rekonstruieren (vgl. z. B. Pachner 2014). Dem liegen auf Selbstreflexion abzielende Texte zugrunde, die von Praktikerinnen und Praktikern im Rahmen des oben beschriebenen Kompetenzanerkennungsverfahrens der Weiterbildungsakademie Österreich verfasst wurden. Diese sogenannten wba-Diplomarbeiten werden mithilfe von Methoden der Grounded Theory (Strauss und Corbin 1996; Charmaz 2014) analysiert. Die Organisation der Auswertungsergebnisse erfolgt mittels MAXQDA12.

Damit der komplexe Zusammenhang zwischen der Nachweisform ,wba-Diplomarbeit" und der Rekonstruktion Reflexiver Kompetenz aus den darin enthaltenen reflexiven Texten nachvollziehbar wird, wird dieser hier am Beispiel eines Einzelfalls veranschaulicht. Dies entspricht nicht zuletzt auch der Betrachtung der zugrundeliegenden Texte in ihrem Anwendungskontext: Dort dienen die in den Diplomarbeiten verschriftlichten Reflexionen zur Bilanzierung der Reflexiven Kompetenz der jeweiligen Verfasserin bzw. des jeweiligen Verfassers. Ausgewählt wird eine Arbeit aus dem Bereich Lehren/Gruppenleitung/Training $(n=14)$. Da der Prozess der Reflexion der Lehrperson nachgezeichnet werden soll, werden Ergebnisse aus dem Auswertungsschritt des axialen Kodierens ausgewählt und der Form des paradigmatischen Modells folgend dargestellt (Strauss und Corbin 1996). Zwar sieht die Grounded Theory Methodology ursprünglich ein fallübergreifendes Vorgehen vor und Modelle des axialen Kodierens begründen sich meist aus mehreren Fällen, doch gibt es auch Studien z. B. aus der Psychologie oder biografieorientierter Forschung, die in einem ersten Schritt einzelfallanalytisch vorgehen und erst in einem nachfolgenden Schritt fallübergreifende Komparationen vornehmen und entlang der Empfehlungen zum Theoretical Sampling Kontrastfälle hinzuziehen (vgl. Mey und Mruck 2009, S. $131 \mathrm{f}$.).

Es handelt sich im Folgenden also um empirische Befunde aus einer sich noch in Arbeit befindenden Studie, um work in progress. Die Nahsicht auf die Analyse eines Einzelfalls dient dazu, das Vorgehen bei der Rekonstruktion zu veranschaulichen und so die daran anschließenden Fallvergleiche vorzubereiten und nachvollziehbar zu machen.

\subsection{Ergebnisse zum Prozess erwachsenenpädagogischer Selbstreflexion - Eine Einzelfallanalyse}

Für die exemplifizierende Einzelfalldarstellung wird der Fall A4 aus dem wba-Diplomschwerpunkt Lehren/Gruppenleitung/Training herangezogen. Darin denkt eine Lehrperson über die Herausforderungen nach, die mit der Durchführung eines Deutschkurses in einem neuen Veranstaltungsformat, der sogenannten Lernwerkstatt, verbunden waren. Der Kurs wurde im Teamteaching mit einer weiteren Lehrperson realisiert. Als besonders herausfordernd erwies sich für die beiden Lehrenden 
der Teilnehmendenwunsch, anders mit der Korrektur von Fehlern umzugehen. Hier mussten sich die zwei Dozierenden auf die Suche nach neuen Wegen und Lösungen machen. Diese Lösungssuche ist Thema der in der wba-Diplomarbeit dazu verschriftlichten (Selbst-)Reflexion.

Als Ergebnis der eingehenden Rekonstruktion dieses Reflexionsprozesses werden im Folgenden Ausschnitte aus dem dazu entlang der Vorgehensweisen der Grounded Theory Methodology entwickelten Theoriememo (Strauss und Corbin 1996; Charmaz 2014) präsentiert. In eckigen Klammern finden sich die Zuordnungen der jeweiligen Befunde zum paradigmatischen Modell (Strauss und Corbin 1996):

Die beiden Kursleiter (KL) sehen sich vehementen und wiederkehrenden Aufforderungen der Teilnehmenden (TN) gegenüber, deren Fehler in der Deutsch-Lernwerkstatt ,auf das Genaueste“ (A4 Abs. 9, 2) zu korrigieren. Sie geraten gleichsam unter Handlungszwang [Ursächliche Bedingungen für die Reflexion]. Zunächst versuchen die beiden Lehrpersonen, nur ausgesuchte Fehler zu korrigieren und zu besprechen, womit die TN aber unzufrieden sind. Sie folgen dann dem Wunsch der TN, alle Fehler zu berücksichtigen, merken aber, dass dies zu Frustration bei allen Beteiligten - TN wie KL - führt [Strategien]. Nach einem offenbar längeren Prozess des Ausprobierens und der iterativen Lösungssuche [Strategien] entwickeln sie die Idee, die Fehler nach „Fehlerphänomenen“ zu gruppieren und nur noch diese „Fehlerphänomen-Gruppen“ (A4 Abs. 10, 2) zu besprechen [Strategie]. Sie lernen also aus Versuch und Irrtum [Strategie]. Fokus der Reflexion ist in diesem Fall das Vermitteln zwischen der Orientierung an den Teilnehmenden und der Motivation (aller) [Phänomen/Fokus der Reflexion]. Die KL agieren dabei im Team und beschränken sich auch bei der Lösungssuche ausschließlich auf dieses Teamteaching. Sie beraten sich nur intern bzw. verzichten umgekehrt darauf, sich Rat von außen zu holen [Strategie]. Das Ausbalancieren zwischen TN-Wünschen und dem Aufrechterhalten der Motivation bei TN wie KL vollzieht sich allein im Lehr-Lernkontext, der Unterrichtssituation an sich [Handlungskontext für die Reflexion]. Dass nicht auf Anhieb eine Lösung gefunden werden konnte, sondern vielmehr eine iterative Lösungssuche [Strategie] unternommen werden musste, verweist darauf, dass es sich offenbar um ein nicht-triviales Problem handelt [Ursächliche Bedingung für die Reflexion]. Als besonders herausfordernd wird zudem die neue Lernumgebung, das neue Lernformat „Lernwerkstatt“ (A4 Abs. 9, 1) beschrieben, in dem die beiden KL ihre „ersten Gehversuche“ (A4 Abs. 9, 1) machen [Ursächliche Bedingungen für die Reflexion]. Warum lassen sich die beiden KL aber überhaupt auf diesen mühevollen und von negativen Gefühlen begleiteten Prozess der Lösungssuche und des Ausbalancierens von TN-Wünschen und Aufrechterhalten der Motivation ein? Offenbar ist ihr Handeln getragen von einer konstruktivistischen Einstellung gegenüber dem Lehren und Lernen (in A4, Abschn. 2, wird explizit darauf rekurriert). Der Konstruktivismus als gegenwärtig leitendes Lehr-Lernparadigma in der Erwachsenenbildung postuliert Teilnehmerorientierung (vgl. z. B. Siebert 2009). Dazu gehört auch, dass die $\mathrm{KL}$ in ihrem Bestreben, eine lernförderliche Lernumgebung zu gestalten und Lernen zu ermöglichen, auf Motivation und Motiviertheit der TN achten [Intervenierende Bedingungen für die Reflexion]. Dass sie dabei auch auf den Erhalt ihrer eigenen Motivation Rücksicht nehmen, kann als Zeichen einer fortgeschrittenen professionellen Kompetenz in Bezug auf die eigene Selbstregulation bzw. 
Motivationalen Orientierungen (vgl. Baumert und Kunter 2006) gewertet werden [Intervenierende Bedingung für die Reflexion]. Für einen hohen Grad professioneller Entwicklung (Novizin vs. Expertin) spricht auch, dass der/die KL in der Lage ist, sein/ihr professionelles, didaktisches Handeln an neue Erfordernisse (hier: ein neues Veranstaltungsformat) flexibel anzupassen [Intervenierende Bedingung für die Reflexion]. Die Anpassung ist nötig und herausfordernd, da der/die KL ursprünglich als „Sprachtrainer/in“ (Selbstbeschreibung in A4, Abschn. 2) eher in eine traditionelle, vermittlungsorientierte Art des Unterrichtens einsozialisiert wurde (vgl. A4, Abschn. 2) [Intervenierende Bedingung für die Reflexion]. Die Vermittlung zwischen Teilnehmerorientierung und Erhalt der Motivation gelingt, indem die Besprechung der sogenannten Fehlerphänomen-Gruppen ,nach und nach [...] fester Bestandteil““ (A4 Abs. 10, 1) des Unterrichts wird [Konsequenz der Reflexion]. Gleichzeitig haben die KL auch ihre Überzeugungen im Umgang mit Fehlern verändert, deren Betrachtung sie nun auch als ,sehr spannend“ (A4 Abs. 9, 4) und damit als Lernchance und nicht mehr nur als störend wahrnehmen und bewerten [Konsequenz der Reflexion]. Dies lässt erste Schlüsse über die Tiefe der Reflexion zu, die hier als eher hoch eingeschätzt werden kann, da sowohl das Unterrichtshandeln als auch darauf bezogene Überzeugungen dauerhaft verändert wurden, was als Hinweis für eine gelungene ergebnisorientierte Selbstreflexion gewertet werden kann (vgl. Greif 2008) [Tiefe der Reflexion].

In Abb. 1 sind die Ergebnisse noch einmal dem paradigmatischen Modell (Strauss und Corbin 1996) entsprechend grafisch dargestellt, wobei unidirektionale Pfeile „daraus folgt/hat Einfluss auf" bedeuten und bidirektionale Pfeile auf einen wechselseitigen Einfluss hinweisen.

Das Modell ist insofern vorläufig, als dass es spätestens beim Einbezug kontrastierender Fälle in weiteren Auswertungsschritten einer Reorganisation unterzogen werden muss.

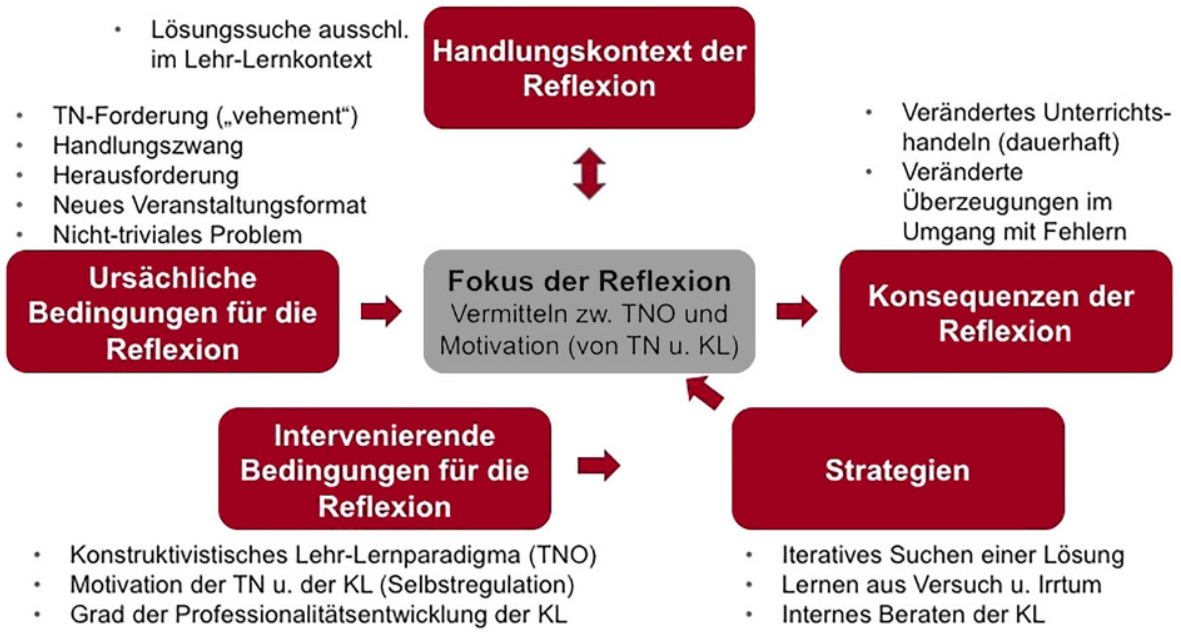

Abb. 1 Paradigmatisches Modell für den Fall „,Vermitteln zwischen Teilnehmerorientierung (TNO) und Motivation“" 


\subsection{Interpretation, Diskussion und Ausblick}

Aus der Nahsicht, die die Darstellung einer beispielhaften Einzelfallanalyse erlaubt, lassen sich insbesondere Hinweise auf den Zusammenhang zwischen der Nachweisform „wba-Diplomarbeit“ und der Rekonstruktion Reflexiver Kompetenz ableiten, aber auch Antworten auf die oben gestellten Fragen für den gewählten Fall finden.

So wird deutlich, dass die im Rahmen der wba-Diplomarbeit verschriftlichten Reflexionen, durchaus das Potenzial haben, verschiedene Aspekte Reflexiver Kompetenz differenziert sichtbar zu machen. Dazu zählen ursächliche Bedingungen für eine Reflexion des eigenen beruflichen Handelns ebenso, wie Prozesse der Lösungssuche und der Umgang mit den erlebten Herausforderungen des Berufsalltages. Unterschiedlich weitreichende Folgen des Nachdenkens über das eigene berufliche Denken und Tun, gerade dort wo Routinen nicht mehr greifen, werden nachvollziehbar. Schließlich lassen sich weniger explizit beschriebene Bedingungen und Kontexte des Handelns, in die die professionell Tätigen eingespannt sind, rekonstruieren. Es deutet sich an, dass das paradigmatische Modell hier einen heuristisch wertvollen Rahmen bietet, um reflexive Prozesse im Kontext professionellen erwachsenenpädagogischen (Lehr-)Handelns zu beschreiben.

Für den beschriebenen Fall kann in aller Kürze festgehalten werden, dass offenbar eine gelungene Reflexion z.B. im Sinne einer ergebnisorientierten Selbstreflexion nach Greif (2008) vorliegt, da das eigene professionelle Handeln in der Folge dauerhaft verändert wird. Ebenso kann konstatiert werden, dass sich hier Professionalität in Form einer professionellen Selbstdeutung und eines entsprechenden Reflexionsstils zeigt, wie Nittel (2000) sie postuliert. Es gelingt den Kursleitenden, die sich auftuenden Differenzen vor allem auf der Ebene des Handelns zu balancieren und nicht einseitig aufzulösen. Auf der Wissensebene dagegen wird wissenschaftliches Wissen etwa zum Umgang mit Fehlern nicht berücksichtigt.

Eine weitere Ausdifferenzierung reflexiver Prozesse entlang des paradigmatischen Modells, wie es die im nächsten Schritt anstehenden Fallvergleiche erlauben, wird zudem einen differenzierteren Blick auf Antinomien, Widerspruchskonstellationen und Paradoxien (vgl. von Hippel 2011; Helsper 2002) zulassen, die Lehrende in der Erwachsenenbildung tagtäglich zu balancieren haben und an denen sich ihre reflexive Kompetenz und ihre Professionalität immer wieder neu erweist.

Wenngleich das Potenzial und der Gehalt selbstreflexiver Texte für die Anerkennung Reflexiver Kompetenzen deutlich wurden, bleibt die Frage, wie im konkreten Anerkennungsalltag mit dieser komplexen Nachweisform umzugehen ist. Hier ist es das Ziel, im weiteren Arbeitsprozess ein Modell zu entwickeln, welches reflexive Prozesse von Lehrenden in der Erwachsenenbildung in ihren zentralen Phänomenen, Bedingungskonstellationen und Strategien so beschreibt, dass sich daraus Kriterien entwickeln lassen für die Anerkennung Reflexiver Kompetenzen. 


\section{Fazit - Reflexive Kompetenzen für den Umgang mit Ungewissheit und Komplexität}

Wie sich gezeigt hat, sind für die Anerkennung Reflexiver Kompetenzen bereits systematisch ausgearbeitete Einzelfallanalysen aufschlussreich. Fallübergreifende Komparationen sind im nächsten Schritt wichtig für die Formulierung und davon abgeleitet die Vermittlung Reflexiver Kompetenzen. Aus ihnen lassen sich nicht nur wichtige Elemente Reflexiver Kompetenz ableiten, sondern sie erlauben es auch einem differenztheoretischen Verständnis von Professionalität folgend, Aussagen über typische Kernprobleme und den gelungenen Umgang mit diesen zu treffen.

Damit weist die Rekonstruktion Reflexiver Kompetenzen über den eingangs geforderten Dreischritt aus Formulierung, Anerkennung und Vermittlung von Kernkompetenzen (Gruber und Wiesner 2012) hinaus. Es lassen sich aus ihr vielmehr Schlussfolgerungen für den gelingenden, reflexiven Umgang mit Ungewissheit und divergierenden oder gar widersprüchlichen Handlungsanforderungen ableiten, dem zentralen Charakteristikum gegenwärtiger, erwachsenenpädagogischer Professionalität (Pachner 2013).

Open Access Dieser Artikel wird unter der Creative Commons Namensnennung 4.0 International Lizenz (http://creativecommons.org/licenses/by/4.0/deed.de) veröffentlicht, welche die Nutzung, Vervielfältigung, Bearbeitung, Verbreitung und Wiedergabe in jeglichem Medium und Format erlaubt, sofern Sie den/die ursprünglichen Autor(en) und die Quelle ordnungsgemäß nennen, einen Link zur Creative Commons Lizenz beifügen und angeben, ob Änderungen vorgenommen wurden.

\section{Literatur}

AK DQR (2011). Deutscher Qualifikationsrahmen für lebenslanges Lernen - verabschiedet vom Arbeitskreis Deutscher Qualifikationsrahmen (AK DQR) am 22. März 2011. https://www.dqr.de/media/ content/Der_Deutsche_Qualifikationsrahmen_fue_lebenslanges_Lernen.pdf. Zugegriffen: 13. Apr. 2018.

Baumert, J., \& Kunter, M. (2006). Stichwort: Professionelle Kompetenz von Lehrkräften. Zeitschrift für Erziehungswissenschaft, 9(4), 469-520.

Cedefop (2015). European guidelines for validating non-formal and informal learning. Luxembourg: Publications Office. Cedefop reference series; No 104. http://dx.doi.org/ https://doi.org/10.2801/ 008370. Zugegriffen: 13. Apr. 2018.

Charmaz, K. (2014). Constructing grounded theory. Los Angeles: SAGE.

Dewe, B. (1990). Pädagogische Handlungskompetenz in Theorie und Praxis der Erwachsenenbildung. Hessische Blätter für Volksbildung, 4/1990, 289-297.

DIE - Deutsches Institut für Erwachsenenbildung (2018). GRETA - kompetent handeln in Training, Kurs \& Seminar. Bonn. https:/www.die-bonn.de/docs/GRETA_Booklet_210x297_4C_rev11_ weboptimiert.pdf. Zugegriffen: 31. Mai 2018.

Erpenbeck, J., \& von Rosenstiel, L. (Hrsg.). (2007). Handbuch Kompetenzmessung. Stuttgart: SchäfferPoeschel.

Greif, S. (2008). Coaching und ergebnisorientierte Selbstreflexion. Göttingen: Hogrefe.

Gruber, E., \& Wiesner, G. (Hrsg.). (2012). Erwachsenenpädagogische Kompetenz stärken. Kompetenzbilanzierung für Weiterbildner/-innen. Bielefeld: W. Bertelsmann.

Helsper, W. (2002). Lehrerprofessionalität als antinomische Handlungsstruktur. In M. Kraul, W. Marotzki \& C. Schweppe (Hrsg.), Biographie und Profession (S. 64-102). Bad Heilbrunn: Klinkhardt.

von Hippel, A. (2011). Programmplanungshandeln im Spannungsfeld heterogener Erwartungen - Ein Ansatz zur Differenzierung von Widerspruchskonstellationen und professionellen Antinomien. REPORT - Zeitschrift für Weiterbildungsforschung, 34(1), 45-60. https://doi.org/10.3278/REP1101W045.

Lencer, S., \& Strauch, A. (2016). Das GRETA-Kompetenzmodell für Lehrende in der Erwachsenen- und Weiterbildung. Bonn: DIE. www.die-bonn.de/doks/2016-erwachsenenbildung-02.pdf. Zugegriffen: 30. Mai 2018. 
Mey, G., \& Mruck, K. (2009). Methodologie und Methodik der Grounded Theory. In W. Kempf \& M. Kiefer (Hrsg.), Forschungsmethoden der Psychologie. Zwischen naturwissenschaftlichem Experiment und sozialwissenschaftlicher Hermeneutik (Bd. 3, S. 100-152). Berlin: Regener.

Nittel, D. (2000). Von der Mission zur Profession? Bielefeld: W. Bertelsmann.

Nittel, D. (2011). Von der Profession zur sozialen Welt pädagogisch Tätiger? Vorarbeiten zu einer komparativ angelegten Empirie pädagogischer Arbeit. In W. Helsper \& R. Tippelt (Hrsg.), Pädagogische Professionalität. Zeitschrift für Pädagogik, Beiheft 57. (S. 40-59). Weinheim: Beltz.

Nittel, D., \& Seltrecht, A. (2008). Der Pfad der „,individuellen Professionalisierung“: ein Beitrag zur kritisch-konstruktiven erziehungswissenschaftlichen Berufsgruppenforschung; Fritz Schütze zum 65. Geburtstag. BIOS - Zeitschrift für Biographieforschung, Oral History und Lebensverlaufsanalysen, 21(1), 124-145. http://nbn-resolving.de/urn:nbn:de:0168-ssoar-270151. Zugegriffen: 24. Aug. 2018.

Pachner, A. (2013). Selbstreflexionskompetenz. Voraussetzung für Lernen und Veränderung in der Erwachsenenbildung? Magazin erwachsenenbildung.at. Das Fachmedium für Forschung, Praxis und Diskurs, 20/2013, S. 06/1-9.

Pachner, A. (2014). Kompetenzen konstruktiv thematisieren: Theoretische Verortung und empirische Rekonstruktion am Beispiel der Selbstreflexionskompetenz. In H. Pätzold, H. von Felden \& S. SchmidtLauff (Hrsg.), Programme, Themen und Inhalte in der Erwachsenenbildung: Dokumentation der Jahrestagung 2013 der Sektion Erwachsenenbildung der Deutschen Gesellschaft für Erziehungswissenschaft (S. 172-189). Baltmannsweiler: Schneider.

Schön, D. A. (1987). Educating the reflective practitioner. San Francisco: Jossey-Bass.

Schütze, F. (1996). Organisationszwänge und hoheitsstaatliche Rahmenbedingungen im Sozialwesen: Ihre Auswirkung auf die Paradoxien des professionellen Handelns. In A. Combe \& W. Helsper (Hrsg.), Pädagogische Professionalität (S. 183-275). Frankfurt a. M.: Suhrkamp.

Siebert, H. (1990). Von der Professionalisierung zur Professionalität? Hessische Blätter für Volksbildung, 4/1990, 283-288.

Siebert, H. (2009). Didaktisches Handeln in der Erwachsenenbildung. Didaktik aus konstruktivistischer Sicht (6. Aufl.). Augsburg: Ziel.

Strauss, A., \& Corbin, J. (1996). Grounded Theory: Grundlagen Qualitativer Sozialforschung. Weinheim: Beltz.

wba - Weiterbildungsakademie Österreich (2016). wba-Diplom: Diplomierte/r Erwachsenenbildner/in Lehren/Gruppenleitung/Training. https://wba.or.at/media/pdf/qualifikationsprofil-wba-diplom-lehrengruppenleitung-training.pdf. Zugegriffen: 30. Mai 2018.

wba - Weiterbildungsakademie Österreich (2018). wba-Zertifikat: Zertifizierte/r Erwachsenenbildner/in. https://wba.or.at/media/pdf/qualifikationsprofil-wba-zertifikat.pdf. Zugegriffen: 30. Mai 2018.

Weinert, F. E. (2001). Leistungsmessungen in Schulen. Weinheim: Beltz. 